\title{
General Parametric Model of The Body of The Total Hip Endoprosthesis
}

\author{
Slobodan Tabaković, Milan Zeljković, Aleksandar Živković \\ Faculty of Technical Sciences, University of Novi Sad \\ Trg D. Obradovića 6, 21000 Novi Sad, Serbia \\ e-mail: tabak@uns.ac.rs,milanz@uns.ac.rs,acoz@uns.ac.rs
}

\begin{abstract}
The endoprostheses of the hip joint are the most commonly used group of implants in orthopedic surgery. The success of their application in the process of replacing natural hip joint with artificial depends, besides the patient related and surgical factors, which include the selection of the type of endoprosthesis and the extent of its adaptability to the patient. Because of that, endoprostheses adapted to the patient's measures, so-called custom made endprostheses, are used. The main challenge in their design is large number of influential factors on the shape and dimensions of the endoprosthesis, the long period of development, and high prices as a consequence of these factors.

One of the directions of endoprosthesis design process improvement is the introduction of generalized computational models that enable the adaptation of the endoprosthesis to a specific patient's femur, taking into consideration all influential factors of the disease.

This paper describes a general parametric model of the body of the hip endoprosthesis, which was developed by combining two methods of parametric modeling with the aim to implement all existing contributing factors and, also, facilitate introduction of the new ones. The main advantages of this model of endoprosthesis are: flexibility and simplicity which makes this model easy for application in a variety of CAD software systems.
\end{abstract}

Keywords: parametric model; endoprosthesis; Bezier curves; Computer Aided Design

\section{Introduction}

Biomechanical researches have shown that some of the bones and joints of the human skeletal system are exposed to considerable strain. This is the result of daily activities in which the average person makes about 10,000 steps [17]. This may, with the influence of various illnesses or injuries, be a source of various degenerative changes in the hip joint elements (Fig. 1), which establish a connection between the upper part of the skeletal system and the femur.

When damage from a disease becomes severe, it is necessary to perform partial or complete (total) hip joint replacement. 


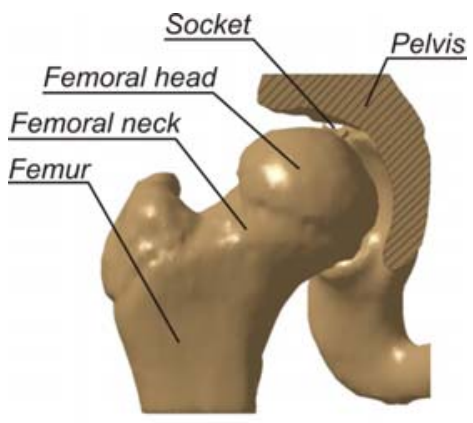

Figure 1

Elements of the hip

The success of the surgery to replace a natural hip joint with the artificial one depends, in addition to the patient related and surgical factors, on so called endoprosthesis factors, which include selection of the type of endoprosthesis and the extent of its adjustment to the patient.

From the design point of view, the primary goal of the endoprosthesis development is to reduce its impact on the success of the surgery. In the last 70 years, since 1938 when the total hip replacement surgery development started [2], a whole range of types of prostheses was developed, which are divided according to their purpose (primary, revision and reconstructive) [7], dimensions (usually there are about 10 models per type) [10], and the way in which the femur is fixed (cement, cementless) [7]. The choice of such standardized endoprosthesis for a patient is made based on the type and complexity of the disease, age of the patient and the basic geometrical parameters of the femur. The main disadvantage of this method of endoprosthesis design is the small number of geometrical factors considered, while some important factors such as the degree of disease and patient ethnicity in general are not considered $[10,16]$.

According to the current research [9, 11], further development of total hip endoprosthesis can be achieved through the development of implants adjusted to the individual characteristics of the patient. In this way, it is possible to include a number of influential factors and achieve the optimum shape and size of the endoprosthesis. The basic prerequisite for this approach is the improvement of methodologies to determine the characteristic geometrical size of the femur $[5,3]$ and improvement of the endoprosthesis design process itself.

The endoprosthesis design phase, in which all influential factors are implemented in the computer model, can be realized by parameterization of endoprosthesis geometry elements and by defining the relationship between influencing factors and geometrical parameters. The particular geometry defined in this way is processed by using parametric modeling CAD software systems. For the purposes of complex modeling, two methods of defining the parametric model are used. 
These are modeling by using of:

- discrete and

- functionally dependent geometrical parameters.

Modeling by using discrete geometrical parameters is the process of modeling of the product geometry, which in the first phase includes the previous system analysis and decomposition of the model geometry of the endoprosthesis to simple geometrical shapes. In the second stage, the individual simple geometrical shapes and their mutual constraints are defined [8]. Integral parts of this model type are discrete parameters given in general numbers that describe geometrical shapes [7].

Previous studies related to the hip endoprostheses design commonly used modeling employing discrete geometrical parameters principle. The main reasons for this were ease of use and the relatively small number of significant factors (mostly geometrical). On the other hand, the biggest disadvantage of parametric models (based on the parameterization process) is the rigid structure that prevents modifying the dependencies between parameters.

The second method, which uses functionally dependent modeling of geometrical parameters, is based on the functional description of mathematical model parameters. It uses mathematical laws that describe: interrelationships between geometrical parameters [14], or spatial areas. For the definition of complex spatial models polynomial and rational Bezier curves ${ }^{1}$ and similar functions are commonly used. This mathematical method of defining the parameters in the model is of a generalized form. This means that the actual endoprosthesis model describes the coordinates of the characteristic points and additional coefficients that have functional dependencies associated with the influential factors in the model. The relationship between the characteristic points and the parameters that define the model is achieved by analytical expressions that may not be contained in the model. Application of functionally dependent geometrical parameters has several advantages over discrete modeling based on geometrical parameters. This primarily includes a significantly more flexible form of the description of geometry, because the model defined is of general nature and can be used for designing different types of endoprostheses. In addition, such models have less geometrical elements that describe the endoprosthesis, which is important from the point of view of speed and accuracy of modeling.

This paper describes the original general parametric model of endoprosthesis body developed by using the functional parametric modeling method which is the result of the previous analysis of geometry of the femur, surgical techniques and a number of existing structural solutions for bodies of the total hip endoprosthesis. Besides the functional dependencies that define geometrical elements of the endoprosthesis body, the specific constraints were additionally introduced in order to simplify the model.

1 Bezier surfaces were developed by Pier Bezier for the Renault car factory in early sixties [12]. 


\section{Materials and Methods}

Design and manufacturing of the total hip endoprosthesis depends on several groups of influencing factors. The most important are: the type and degree of illness, the elements of the femur geometry and the characteristics of surgical techniques used in replacement of a natural with an artificial hip joint. Among them, the largest group consists of geometrical quantities describing the geometry of the femur and its location in the skeletal system, which is important to assure that the patient can walk correctly and smoothly after surgery.

From the design point of view, the endoprosthesis body (femoral stem), as the most complex element of the hip joint endoprosthesis, is a geometrical entity that globally has the same elements. This is the main motivation for the introduction of a unique geometrical form that describes the various forms of body endoprosthesis. This form, a general parametric model of the endoprosthesis body, is a series of interconnected mathematical laws that describe the geometry of the endoprosthesis through the implementation of all influencing factors.

\subsection{The Geometry of the Femur}

The femur is the longest and, according to its mechanical properties, the strongest bone in human body [10]. Its geometrical properties can be viewed through the position and shape of the exterior and / or interior surfaces. The most important elements of the upper half of the femur (important for the design of endoprosthesis) are (see Fig. 2) [16]: Head (1), Neck (2), Trochanter greater (3), Trochanter lesser (4), the body of the femur (corpus femoris) (5) and femoral (or medullary) channel (6)

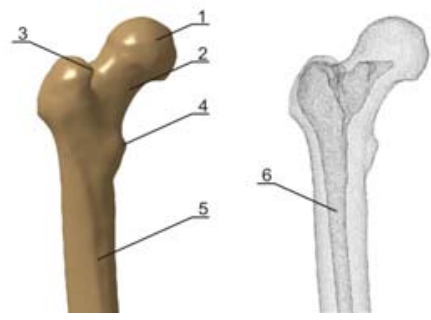

Figure 2

Femur geometry elements

In the anthropometry of the lower extremities of the human body, a group of geometrical values that are influential factors in the design of endoprosthesis [9] are used to describe the position and size of individual elements of the femur, as well as its position in the skeleton. Due to the position of the femur in the skeletal system, these values are described in characteristic anatomical planes: axial (Ap), coronal (Cp) and sagittal (Sp) (Fig. 3). 


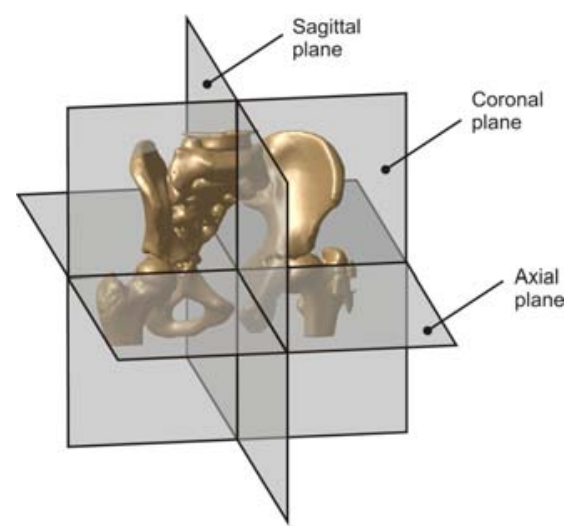

Figure 3

Basic anatomical planes

The characteristic values in the coronal plane are (Fig. 4):

- The angle of inclination of the femur body

- $\quad$ The length of the femoral neck (P)

- Position of the center of femoral head

- The distance between the center of the femoral head and the femur axis $\left(\mathrm{A}_{1}\right)$

- $\quad$ The diameter of the femur head (B)

- Distance between the femoral head from the lesser trochanter (C)

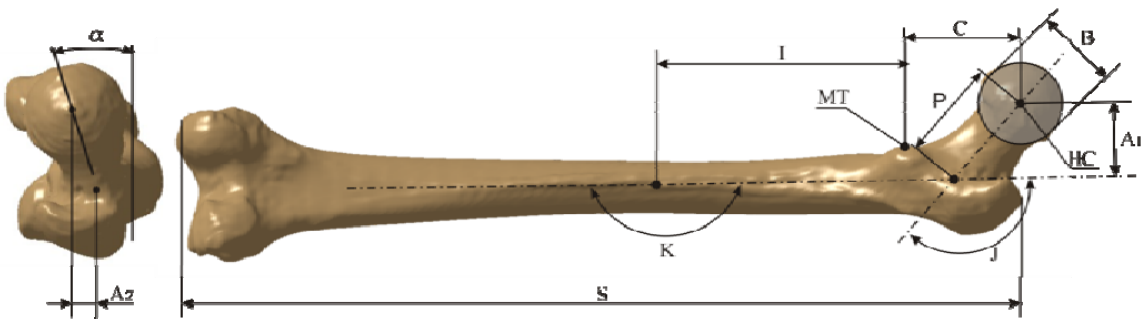

Figure 4

Influential factors in the coronal plane

Another group of influential factors, originating from the geometry of the femur, is defined in the axial plane. It includes:

- Anteversion, the angle between the axis of the femoral neck and coronal planes $-\alpha$ (Figure 4)

- $\quad$ Position of the center of the femoral head $\left(\mathrm{A}_{2}\right)$ 
Among the geometrical values which determine the shape and dimensions of the internal geometry of the femur i.e. the medullary channel, the most commonly used are as follows (Fig. 5):

- The position of the medullary channel isthmus $(\mathrm{G})$

- The expansion coefficient of the medullary channel (Channel flare index $\mathrm{CFI}$ ), which is determined by the quotient of the channel width in the planes that are positioned in the coronal plane, $20 \mathrm{~mm}$ below (F) and above (D) of the lesser trochanter, equation (1) [12].

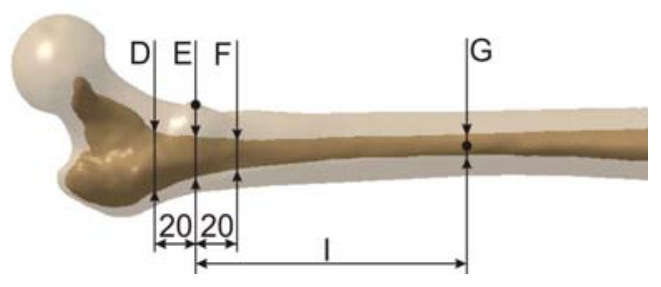

Figure 5

The influential factors of the internal geometry of the femur

$C F I=D / F$

The above geometrical quantities determine the position that the endoprosthesis should have in the femur, the global dimensions of endoprosthesis individual body segments and their mutual relationship. As such, they make the basis for a detailed sizing of certain segments of the endoprosthesis.

In addition to the basic geometrical factors, usage of the diagnostic devices based on tomography methods of recording allows introduction of additional factors. They are consequence of the medullary channel shape and influence the process of surgical procedure. These are:

- Dimensions of the cross sections in several characteristic planes (or explicit mathematical expression ) ( $\mathrm{d}_{\mathrm{MCAP}}, \mathrm{d}_{\mathrm{MCLM}}$ on Fig. 6), [12]

- Cement layer thickness (S on Fig. 6).

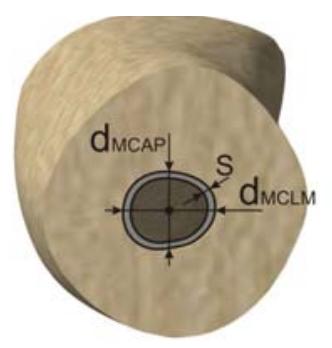

Figure 6

The cross-section of the femur 


\subsection{Appearance and Endoprosthesis Body Elements}

As a result of endoprosthesis body shape standardization, for many years, there are categories of hip joint endoprostheses that are grouped according to the type of disease and the fixation mode of the endoprosthesis into the femur. This approach is verified by the ISO 7206-1/2008 [7], so that endoprostheses are categorized into following types:

- Primary, intended for installation in cases of femurs with osteoarthritis disease [6],

- Revision, used for installation as a replacement for primary endoprostheses, [13]

- Reconstruction, used for severe bone diseases

Regardless of the categorization, all types of hip endoprostheses have the same geometrical segments, defined by a series of surfaces. These segments have a specific purpose in the endoprosthesis. Fig. 7 shows the structures of the endoprosthesis body with distinctive segments.

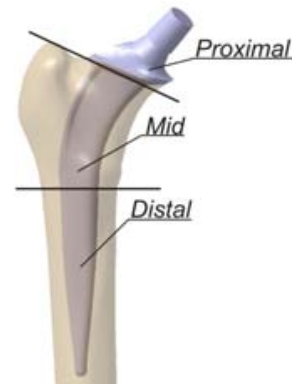

Figure 7

Geometrical segments of the femoral prosthesis body

As it can be seen in Fig. 7, the body of the endoprosthesis has, considering the form and function, three segments:

- distal, which allows the positioning of endoprosthesis body in the medullary channel of the femur

- $\quad$ mid, segment, which passes through the porous part of the bone and follows the anatomical parameters of the femur,

- proximal, which contains an artificial femoral neck and the collar.

Each of these segments is described by one or more surfaces, the shape and dimensions of which depend on several influential factors. 


\subsection{Geometrical Factors Influencing the Total Hip Endoprosthesis}

Reconstruction of the bone geometry, which is the basis for defining the geometrical factors required for the design of hip prostheses, involves the use of several pieces of topological information obtained mostly by using diagnostic devices. In addition to analog X-ray based devices, in medical diagnosis usually digital devices based on tomography methods of recording and computerized tomography (CT) or magnetic resonance imaging (MRI) are used. These digital devices deliver a series of images of diseased limbs cross sections at appropriate planes.

By mathematical processing of digital information represented with the diagnostic image, their conversion is done to the form which allows the formation of CAD models of the femur [18]. These models can be in the form of point clouds or complex volumes obtained by voxelization (replacing pixels as the basic element of the image with voxels) [20].

The basic advantage of reconstruction of the femur on the basis of tomography images is the possibility for precise determination of a large number of characteristic points of the external geometry of the medullary channel of the femur. Geometrical factors influencing the femur, defined on the basis of this information, are used in the design of one or more segments of the endoprosthesis.

The distal segment of the endoprosthesis body is the geometrical form which depends on the shape and dimensions of the medullary channel of the femur. For definition of this segment the following parameters are used:

- The position of the medullary channel isthmus

- Lesser trochanter position

- Layout and dimensions of the medullary channel.

The mid segment of the endoprosthesis body provides its proper positioning, enables the transfer of the load from the pelvic region to the foot and ensures its proper position relative to the rest of the body. The basic geometrical elements that define the shape and dimensions of the middle segment of the endoprosthesis are:

- Position of the lesser trochanter

- Position of the greater trochanter

- The appearance and dimensions of the medullary channel in axial cross-sections $20 \mathrm{~mm}$ above and below the lesser trochanter

- The angle of inclination of the body of the femur

- Femoral anteversion angle

- CFI expansion coefficient of the medullary channel 
The proximal segment consists of four parts: a body, collar, neck and cone upon which the artificial femoral head is placed. The dimensions and shape of the first two parts are mostly determined for the family of endoprostheses, based on structural and exploitation conditions defined by the manufacturers. The neck and artificial femoral head should ensure proper placement of the body in conjunction with the remaining elements of the endoprosthesis and provide the proper movement of the patient after the hip replacement surgery. The most important geometrical dimensions of the elements that determine these segments are: the position of the femoral head, the distance of the femoral head from the axis, the angle of the femoral neck.

Analysis of hip joint diseases treated by THR method and geometrical parameters necessary to define the existing types of prostheses [7] indicate that the number of influential factors resulting from the femur geometry varies.

\subsection{Application of Rational Bezier Curves in Modeling of the Endoprosthesis Body}

In computer technology there is a number of mathematical methods that are used to describe complex surfaces. Among them, an important role is played by the rational Bezier curves (Equation 2) belonging to the family of curve surfaces based on the generalized description of the parameter defined polynomial curves.

$$
C(t)=\frac{\sum_{i=0}^{n} B_{i}^{n}(t) w_{i} P_{i}}{\sum_{i=0}^{n} B_{i}^{n}(t) w_{i}}
$$

The elements of the equations of these curves are:

- Control points $\left(\mathrm{P}_{\mathrm{i}}\right)$ which determine the degree of the polynomial and bounds propagation curves

- Elements of the Berstein polynomial $B_{i}(t)$, described by equation 3 and

$$
B_{i}^{n}(t)=\left(\begin{array}{c}
n \\
i
\end{array}\right)(1-t)^{n-i} t^{i}, \mathrm{i}=0,1,2, \ldots \mathrm{n}, \mathrm{t} \in[0,1]
$$

- Weighting factors $\mathrm{w}_{\mathrm{i}}$ regulating the convergence degree of the curve to control points. Fig. 8 shows the impact of changing weighting factor $\mathrm{w} 2$ to the shape of the curve around that point. 


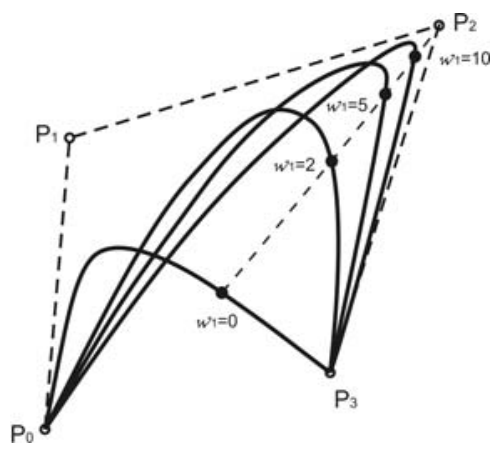

Figure 8

The influence of the weighting factor $\mathrm{w}_{2}$ to the shape of the rational Bezier curve

Primary advantages of the application of rational Bezier curves in defining the geometry of the computer model are: the ability to correct the curve by using the weighting factor and the possibility of forming an explicit mathematical expression for the curve of a known degree.

By using the same procedure, in addition to spatial curves, we are introducing the concept of rational Bezier surfaces (or the tensor defined surfaces) determined by specific control points $(\mathrm{Pi}$ and $\mathrm{Pj})$, by Berstein polynomials $\mathrm{Bi}(\mathrm{t})$ and $\mathrm{Bj}(\mathrm{t})$, where $\mathrm{t} \in[0,1]$ and the weighting factor wi,j (Equation 4).

$P(t)=\frac{\sum_{i=0}^{n} \sum_{j=0}^{m} B_{i}^{n}(t) B_{j}^{m}(t) w_{i j} P_{i} P_{j}}{\sum_{i=0}^{n} \sum_{j=0}^{m} B_{i}^{n}(t) B_{j}^{m}(t) w_{i j}}$

Application of Bezier surfaces allows definition of surface models of objects of arbitrary complexity.

\section{General Parametric Model of the Endoprosthesis Body}

The general parametric model of the endoprosthesis body is a structure composed of geometrical elements described by functional and discrete parametric models. The main characteristic of the general model is the ability to implement all mentioned influential factors that are necessary for the description of the endoprosthesis body and a possibility to add the new ones. In this specific case, the model is based on the two parts that have a different role in the endoprosthesis. These are:

- the femoral part that contains the distal and mid segment of the endoprosthesis is defined by rational Bezier function and 
- the proximal part that consists of the proximal segment is defined by the three, relatively simple, geometrical shapes.

The femoral part includes segments of the endoprosthesis body (the distal and mid segment) that are located within the femur after the installation. It is specified by a number of parameters, resulting from the geometry of the femur, and a series of exploitation demands arising from the operational method used and the need for increased stiffness of the femur and endoprosthesis body assembly [1, 4] (Fig. 9).

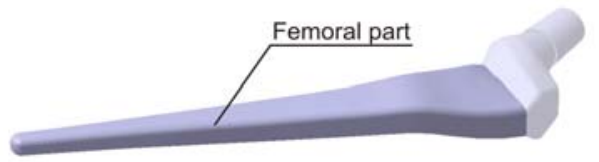

Figure 9 Femoral part of endoprosthesis body

Bezier surface describing the femoral part of endoprosthesis body is formed by combining the two planar Bezier functions in the characteristic planes of the femur (the axial and coronal plane).

In the axial plane, in which the cross-section of the endoprosthesis body is defined, the complexity of the medullary channel and the number of significant factors that are taken into account, are the reason why the curve is determined by Bezier planar function of the tenth degree. Due to better control of the parameters that determine the control points, the curve sections are arranged in the schematic diagram shown in Fig. 10, where they are defined by the distance from the horizontal and vertical axes. This description of the cross-section provides definition of endoprostheses with the symmetric as well as with the asymmetric cross-section.

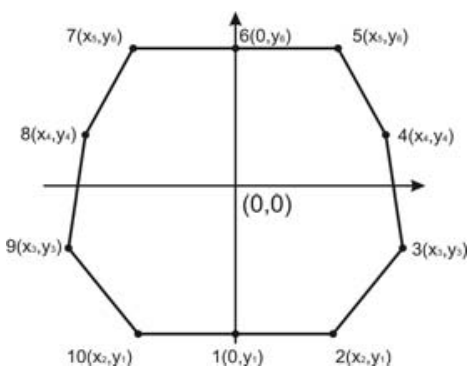

Figure 10

Schematic layout of control points of the endoprosthesis body cross-section

As it is shown on the previous figure, some points are mutually interrelated with the constraints in values of coordinates. This was introduced in order to reduce the degree of uncertainty of the system of equations which determine the parameters of Bezier surfaces, bearing in mind that they are defined on the basis of the influential factors on the body of the endoprosthesis. 
The implementation of these points in the curve equation a function of the endoprosthesis cross-section is obtained expressed by a Bezier curve [12].

$$
A(t)=\frac{\sum_{i=0}^{10} B_{i}^{10}(t) w_{i} P_{i}}{\sum_{i=0}^{10} B_{i}^{10}(t) w_{i}}
$$

As noted above, for defining the geometry of the endoprosthesis body in coronal plane along the distal segment, the two characteristic points are necessary (the midpoint of the medullary channel at the narrowest part and $20 \mathrm{~mm}$ below the lesser trochanter). Additionally, the mid segment is determined with the three points (the midpoint of medullary channel $20 \mathrm{~mm}$ below the lesser trochanter, 20 $\mathrm{mm}$ above it and in the plane in which the femoral neck is surgically removed). On the basis of this, it was concluded that the generators of the endoprosthesis can be described by four characteristic points (the plane $20 \mathrm{~mm}$ below the lesser trochanter is a place where the distal and middle segments are connecting), so the Bezier curve in the coronal plane can be expressed as follows:

$$
B(t)=\frac{\sum_{j=0}^{4} B_{j}^{4}(t) w_{j} P_{j}}{\sum_{j=0}^{4} B_{i}^{4}(t) w_{j}}
$$

On the basis of the existing partial equations a Bezier surface function can be imported, which includes the aforementioned curves and allows the definition of endoprosthesis segments located within the femur.

$$
P(t)=\frac{\sum_{i=0}^{10} \sum_{j=0}^{4} B_{i, p}^{10}(t) B_{j, p}^{4}(t) w_{i j} P_{i} P_{j}}{\sum_{i=0}^{10} \sum_{j=0}^{4} B_{i, p}^{10}(t) B_{j, p}^{4}(t) w_{i j}}
$$

Fig. 11 shows an example of the femoral endoprosthesis described by the abovementioned equations.

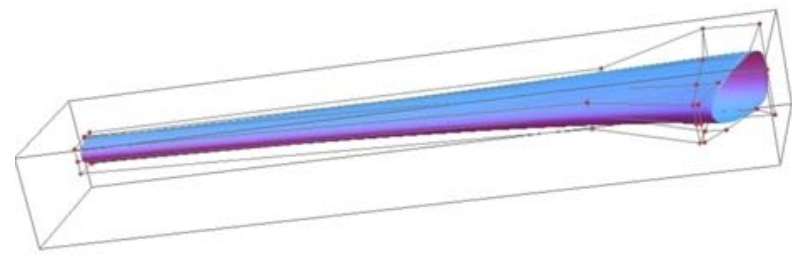

Figure 11 The example of the mathematical description of the femoral part

The proximal segment of the endoprosthesis body is structurally simpler segment of the endoprosthesis body. It contains a series of standardized elements which provide reliance of the endoprosthesis on the femur, and provide the proper formation of the artificial hip joint. These are (Fig. 12):

- the body of proximal segment $\left(\mathrm{T}_{\mathrm{ps}}\right)$ whose geometry depends on the type of endoprosthesis, 
- the artificial femoral neck $\left(\mathrm{N}_{\mathrm{ps}}\right)$, which is usually in the form of cone and

- the tapered connection element between the body and endoprosthesis artificial femoral head (usually a cone with slope 1:10 or Morse taper $\left(\mathrm{T}_{\mathrm{ps}}\right)$ ).

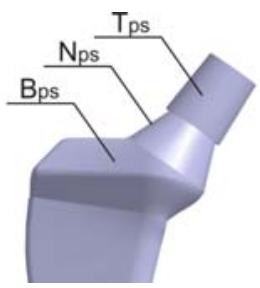

Figure 12

The proximal segment of the endoprosthesis

Because of the simple geometry and a small number of influential factors that determine it, this segment is in the general parametric model implemented as a whole, described by geometrical shapes based on discrete parameters. Fig. 13 shows the final model of the endoprosthesis body obtained by applying a general parametric model.

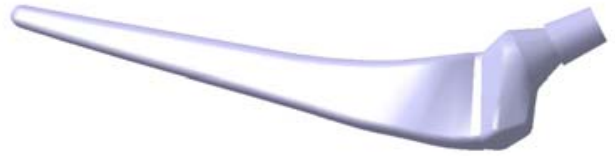

Figure 13

The hip joint endoprosthesis body

\section{Results}

In order to verify the general parametric model of the hip endoprosthesis body, a geometrical modeling for different types of endoprostheses that are in clinical use was done.

As the first phase, a verification of the segment of the general parametric model, used to define the geometry of the intersection of the endoprosthesis was carried out. This was realized by defining several characteristic cross-sections that are used for the body of the endoprosthesis. Fig. 14 shows the cross-sections of the most commonly used types of hip joint endoprosthesis body [15]. 


\section{8
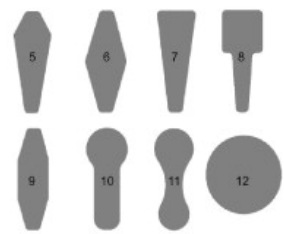

Figure 14

Cross-sections of different endoprosthesis body types [15]

The research [15] has indicated that, from those shown, the most common crosssections used in practice include: circular (especially in tumors and total prostheses), trapezoidal and combined shape.

Based on the analysis of the frequency of their application, the four endoprosthesis profiles were chosen: round, rectangular, trapezoidal, and combined ("mushroom like"), and curves were defined for them based on real dimensions of the crosssection of the femur. Fig. $15 \mathrm{a}, \mathrm{b}, \mathrm{c}$ and $\mathrm{d}$ shows the cross-sections of the endoprosthesis body described by Bezier functions.

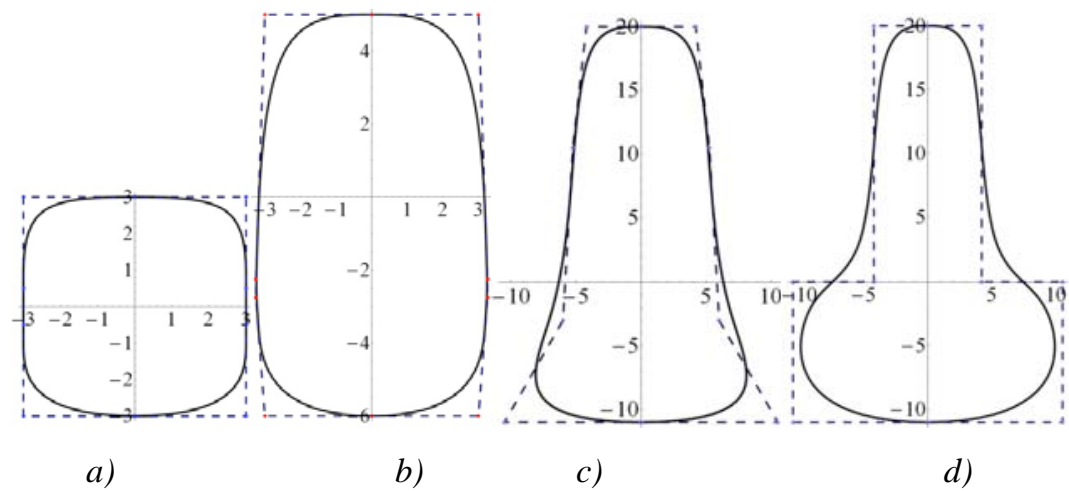

Figure 15

Mathematical interpretation of the endoprosthesis body cross-section

Table 1 shows the list of matrices containing the coordinates of control points for real dimensions of the analyzed sections. 
Table 1

Coordinates of control points

\begin{tabular}{|l|l|llllllllll|}
\hline Type of curve & \multicolumn{10}{|c|}{ Matrix of coordinates of points } \\
\hline Cross-section a & {$\left[\begin{array}{ccccccccccc|}0 & 3 & 3 & 3 & 3 & 0 & -3 & -3 \\
3 & -3 & -.5 & .5 & 3 & 3 & 3 & .5\end{array}\right]$} & & & & \\
\hline Cross-section b & {$\left[\begin{array}{cccccccccc}0 & 3 & 3.25 & 3.25 & 3 & 0 & -3 & -3.25 & -3.25 & -3 \\
-6 & -6 & -2.75 & 2.25 & 5 & 5 & 5 & -2.25 & -2.75 & -6\end{array}\right]$} \\
\hline Cross-section c & {$\left[\begin{array}{cccccccccc}0 & 10.5 & 6 & 5.25 & 4.2 & 0 & -4.2 & -5.25 & -6 & -10.5 \\
-11 & -11 & -3 & 10.5 & 20 & 20 & 20 & -10.5 & -3 & -11\end{array}\right]$} \\
\hline Cross-section d & {$\left[\begin{array}{cccccccccc}0 & 10.5 & 10.5 & 4.2 & 4.2 & 0 & -4.2 & -4.2 & -10.5 & -10.5 \\
-11 & -11 & 0 & 0 & 20 & 20 & 20 & 0 & 0 & -11\end{array}\right]$} \\
\hline
\end{tabular}

To reproduce these curves it is important to note that here the weighting factor 1 (one) was used for each characteristic point. By increasing the weighting factors the profile can be adjusted to the control points.

In the second stage of verification, the endoprosthesis body modeling for the three types of clinically tested, and for several years exploited, endoprostheses was done. These are:

- The body of the primary hip endoprosthesis BB2 (manufactured by DES Novi Sad - Serbia);

- The body of anatomical endoprosthesis BB3 (developed by the Faculty of Technical Sciences in Novi Sad in cooperation with the Institute of Orthopedic Surgery Banjica, Serbia) with the anteversion in the middle part;

- Body of the tumor endoprosthesis (manufacturer Grujic \& Grujic, Serbia).

For verification purposes, and the further studies, the general parametric model is implemented in a software system CATIA. Modeling was realized in two phases where the spatial surfaces of the femoral part were formed, which, after conversion into a volume, were integrated with the proximal segment of the endoprosthesis. Fig. 16 shows a model of the femoral endoprosthesis BB2 defined by Bezier curve surfaces in Wolfram Mathematica software system (Fig. 16a), the same model defined in CATIA software system with the additional ribs (Fig. 16b), as well as photo of the body of endoprosthesis to which an element in the form of ribs was added to increase rigidity (Fig. 16c). 


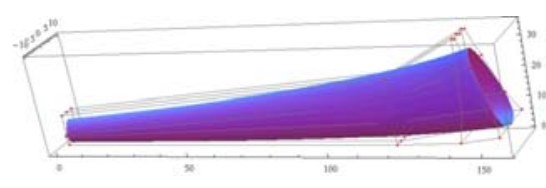

a)

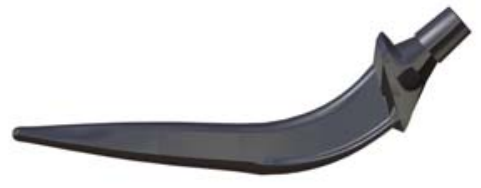

b)

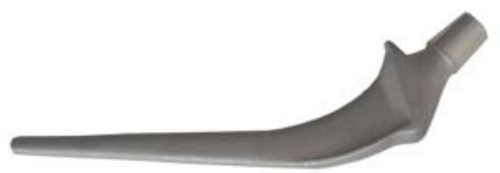

c)

Figure 16

Mathematical and CAD model and a photograph of the femoral endoprosthesis BB2

The second type of endoprosthesis which was modeled is geometrically more complex and belongs to the group of so-called anatomical endoprostheses containing anteversion. From this point of view this type of endoprosthesis is more complex for modeling. Fig. 17 presents a model of the femoral endoprosthesis BB3 defined by Bezier curve surfaces in Wolfram Mathematica software system (Fig. 17a), its interpretation in the CATIA software system (17b) and a photo of the endoprosthesis (17c).

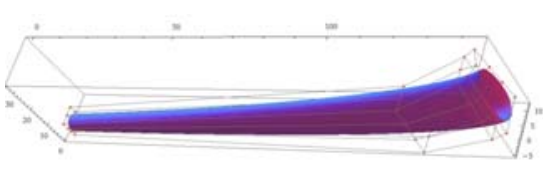

a)

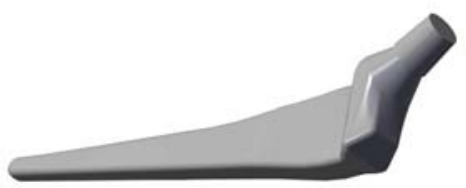

b)

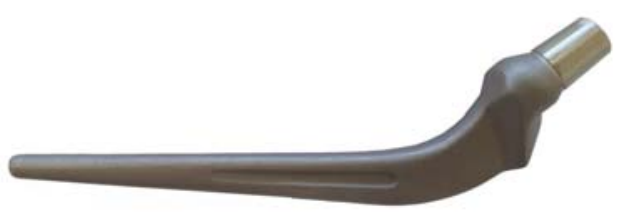

c)

Figure 17

Mathematical and CAD model and a photograph of the femoral endoprosthesis BB3

Finally, the last type of endoprosthesis which was modeled was a total endoprosthesis of the hip joint, which is used to treat tumors (sarcomas)[19]. This type of endoprosthesis is geometrically the simplest because it consists of mostly 
conical elements. This means that the femoral part is defined as a cone that is smaller in length than other types of prostheses, since the surgery removes most of the femur. Fig. 18 presents the femoral part, as described in the Mathematica software system (18a) and CATIA (18b) and a corresponding photo (18c).

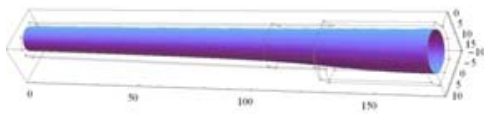

a)

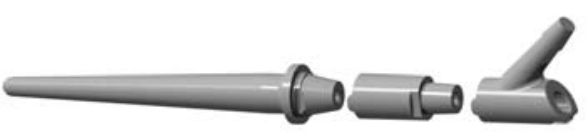

b)

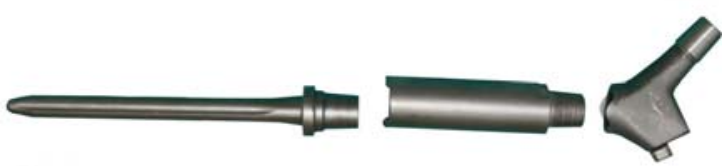

c)

Figure 18

Mathematical and CAD model and a photograph of the femoral part of tumor endoprosthesis BB3

\section{Discussion}

The research results presented in this paper can be observed from the two perspectives: the analysis of the applicability of the general parametric model of the endoprosthesis body, and through the results of verification.

Based on the description and interpretation of the structure of the general parametric model (especially based on the Fig. 15), it can be concluded that the presented model can be applied only in the design of prostheses which is symmetric with one of the axes in the cross section plane. This is a consequence of the aim to minimize the number of variables that occur in the design of endoprostheses. On the other hand, the analysis of current endoprostheses solutions suggests that in clinical practice endoprostheses with the asymmetrical cross section are not used. In this way, a mathematical interpretation of the endoprosthesis body model is obtained so that approximately the same number of geometrical parameters (point coordinates) is used as in the case of models with discretely defined parameters. The basic advantage of this model is significantly increased flexibility, which is reflected in the possibility to adapt the shape of the endoprosthesis to the medullary channel of the femur, as well as introduction of one generic model for all standardized forms of endoprosthesis body. 
Verification presented in this paper was carried out to confirm the ability of the general geometrical model to conform to the shape and profile of the existing forms of enodprostheses. Since the aim of introducing a general model of the design of this product is to promote the development of custom-made prostheses that can be adjusted to the specific femur, a more detailed verification with approximation of errors of described shapes is not applicable here. Further improvement of the general model should include an analysis of the possibilities to approximate forms of medullary channel while taking into consideration other influential factors.

\section{Conclusions}

Designing the hip joint endoprostheses has, for many years, been one of the interesting areas of scientific research activities, due to the large number of influencing factors and complex geometry.

Available results indicate that there are two directions of endoprosthesis design improvement. The first direction is related to a research aimed at improving the characteristics of the endoprosthesis by introducing new influential factors obtained by simulation of the behavior of the endoprosthesis body as a result of FEA analysis, experiments and clinical monitoring of recovery process in patients. The second direction is related to the research targeted at improving the design process to allow creation of the endoprosthesis with measures adjusted to a specific patient, over a short period of time and at a reasonable price. From the engineering point of view these two contradictory demands can be met by increasing the flexibility of computer models and with their structural simplification, based on which the development of CAD systems with the partial automation of the design process becomes possible.

This paper describes the structure of an original general parametric model of the hip endoprosthesis body, adapted to the observed endoprosthesis improvement directions. The main advantages of general parametric model usage are the increased flexibility of the model as well as the model structure that allows calculation of the geometrical parameters independently of the CAD program used to realize the modeling. In this way, a basis for a fully efficient practical automation of some phases of the endoprosthesis design process is created.

\section{References:}

[1] P. Benum, A. Aamodt, "Uncemented Custom Femoral Components in Hip Arthroplasty A Prospective Clinical Study of 191 Hips Followed for at Least 7 Years", Acta Orthopaedica, Vol. 81, pp. 427-435, 2010

[2] J. J. Callaghan, A. G. Rosenberg, H. E. Rubash, "The Adult Hip", Lippincott Williams \& Wilkins, 1997

[3] P. Cerveri, M. Marchente, W. Bartels, K. Corten, J. P. Simon, A. Manzotti, "Automated Method for Computing the Morphological and Clinical Parameters of the Proximal Femur Using Heuristic Modeling Techniques", 
Annals of Biomedical Engineering, Vol. 38, pp. 1752-1766, 2010

[4] R. D. Crowninshield, R. A. Brand, R. C. Johnston, J. C. Milroy, "An Analysis of Femoral Component Stem Design in Total Hip Arthroplasty", The Journal of bone and joint surgery. American volume, Vol. 62, pp. 6878,1980

[5] X. Dong, G. Zheng, "Fully Automatic Determination of Morphological Parameters of Proximal Femur from calibrated Fluoroscopic Images through Particle Filtering", Proceedings of the Third international conference on Image Analysis and Recognition, Vol. II, Springer-Verlag, Portugal, pp. 535-546, 2006

[6] N. P. Hailer, G. Garellick, J. Karrholm, "Uncemented and Cemented Primary Total Hip Arthroplasty in the Swedish Hip Arthroplasty Register Evaluation of 170,413 operations", Acta Orthopaedica, Vol. 81, pp. 34-41, 2010

[7] ISO 7206-1-2008, "Implants for Surgery - Partial and Total Hip Joint Prostheses - Part 1: Classification and Designation of Dimensions", International Organization for Standardization, Geneva, Switzerland, 2008

[8] Y. Jun, K. Choi, "Design of Patient-Specific Hip Implants Based on the 3D Geometry of the Human Femur", Advances in Engineering Software, Vol. 41, pp. 537-547, 2010

[9] Y. Jun, "Morphological Analysis of the Human Knee Joint for Creating Custom-Made Implant Models", International Journal of Advanced Manufacturing Technology, Vol. 52, pp. 841-853, 2011

[10] Y. Kalairajah, S. Molloy, M. Patterson, "The Effect of Femoral Stem Size on Failure Rates in Total Hip Replacement Cemented with Boneloc", Acta orthopaedica Belgica, Vol. 68, pp. 33-36, 2002

[11] K. Kawate, Y. Ohneda, T. Ohmura, H. Yajima, K. Sugimoto, Y. Takakura, "Computed Tomography-based Custom-Made Stem for Dysplastic Hips in Japanese Patients", Journal of Arthroplasty, Vol. 24, pp. 65-70, 2009

[12] H. J. Laine, M. Lehto, T. Moilanen, "Diversity of Proximal Femoral Medullary Canal", Journal of Arthroplasty, Vol. 15, pp. 86-92, 2000

[13] H. Malchau, L. Ahnfelt, "Prognosis of Total Hip Replacement in Sweden: Follow-Up of 92,675 Operations Performed 1978-1990", Acta Orthopaedica Scandinavica, Vol. 64, pp. 497-506, 1993

[14] D. Patel, T. Goswami, "Influence of Design Parameters on Cup-Stem Orientations for Impingement Free RoM in Hip Implants", Medical Engineering \& Physics, Vol. 34, pp. 573-578, 2012

[15] A. Ramos, A. Completo, C. Relvas, J. A. Simoes, "Design Process of a Novel Cemented Hip Femoral Stem Concept", Materials \& Design, Vol. 33, pp. 313-321, 2012 
[16] B. R. Rawal, R. Ribeiro, R. Malhotra, N. Bhatnagar, "Design and Manufacturing of Femoral Stems for the Indian Population", Journal of Manufacturing Processes, Vol. 14, pp. 216-223, 2012

[17] B. B. Seedhom, N. C. Wallbridge, "Walking Activities and Wear of Prostheses", Annals of the Rheumatic Diseases, Vol. 44, pp. 838-843, 1985

[18] W. Sun, B. Starly, J. Nam, A. Darling, "Bio-CAD Modeling and its Applications in Computer-aided Tissue Engineering", Computer-Aided Design, Vol. 37, pp. 1097-1114, 2005

[19] S. Tabakovic, A. Zivkovic, J. Grujic, M. Zeljkovic, "Using CAD/CAE Software Systems in the Design Process of Modular, Revision Total Hip Endoprosthesis", Academic Journal of Manufacturing Engineering AJME, Vol. 9, pp. 97-102, 2011

[20] D. J. Yoo, "Three-Dimensional Surface Reconstruction of Human Bone Using a B-Spline-based Interpolation Approach", Computer-Aided Design, Vol. 43 pp. 934-947, 2011 